\title{
Editorial
}

\section{Aortic stenosis and angina with normal coronary arteries: the role of coronary flow abnormalities}

\begin{abstract}
Angina is a common symptom among patients with significant aortic valve stenosis. The incidence of between $60 \%$ and $80 \%$ is notably higher than that observed in other valve diseases. ${ }^{12}$ In addition, a considerable proportion of patients with angina and aortic stenosis are found to have normal coronary arteries at angiography despite having objective evidence of myocardial ischaemia on electrocardiographic or radionuclide evaluation ${ }^{3}$ and, more recently, stress echocardiography. ${ }^{5}$ Why should aortic stenosis provoke angina in patients without significant obstructive coronary artery disease? The presence of left ventricular hypertrophy undoubtedly plays a role, but recent Doppler echocardiographic studies of the coronary vasculature in aortic stenosis have demonstrated abnormal phasic flow profiles that may shed light on the mechanism of ischaemia in this condition.
\end{abstract}

\section{Role of left ventricular hypertrophy}

The development of left ventricular hypertrophy has been shown to produce considerable impairment of coronary flow reserve in a variety of conditions associated with chronic pressure overload, including aortic valve disease. ${ }^{6}$ Reduced flow reserve is known to provoke ischaemia in these conditions and has been cited as the possible cause of angina in aortic stenosis. ${ }^{7}$ However, it is well known that replacement of the aortic valve relieves angina in the immediate postoperative period before any significant change in the degree of hypertrophy, indicating that impaired flow reserve is not the whole story. Myocardial wall tension (and thus myocardial oxygen demand) is increased and coronary perfusion pressures decreased in aortic stenosis; both will be ameliorated immediately following valve replacement when the obstruction to left ventricular outflow is relieved. Thus an imbalance between oxygen supply and demand also seems likely to be implicated in the genesis of ischaemia in this condition.

\section{CORONARY FLOW RESERVE IN AORTIC STENOSIS}

Resting epicardial coronary artery blood flow has been shown to be increased in patients with aortic stenosis. ${ }^{8}$ In addition, Villari $e t a l^{9}$ have shown that the left (but not the right) coronary artery cross-sectional area is significantly greater in patients with aortic stenosis and left ventricular hypertrophy compared with controls without hypertrophy. These findings are likely to reflect an attempt by the coronary vasculature to keep up with the increasing metabolic demand of the hypertrophied left ventricle. Indeed, when coronary flow is indexed to unit left ventricular mass in this situation, resting indexed flow is in fact normal. ${ }^{8}$ Similarly at the outset of Villari's study when left coronary artery area was indexed per $100 \mathrm{~g}$ of left ventricular muscle mass, there was no difference between indexed left coronary artery area in patients with aortic stenosis and controls. However, as the degree of hypertrophy increased during the course of that study, the indexed left coronary artery area became significantly reduced in the aortic stenotic group. These findings present an interesting hypothesis: that the epicardial coronary vasculature, while initially adapting to produce higher rates of blood flow to the hypertrophied myocardium in aortic stenosis, ultimately fails to keep pace as the degree of hypertrophy worsens. This concept is further supported by the findings that during vasodilator stress maximal indexed coronary flow and the maximal/resting flow ratio (that is, coronary flow reserve) are significantly reduced and indexed coronary artery resistance increased in aortic stenosis. ${ }^{810}$ The hypertrophied ventricle is thus rendered susceptible to ischaemia under conditions of increased workload, even in the absence of significant coronary artery stenoses.

\section{Phasic coronary flow in aortic stenosis}

Coronary flow reserve calculations are typically derived from flow catheters positioned in the coronary sinus. This technique, however, provides little meaningful information regarding phasic flow across the left ventricular vasculature during systole and diastole. With recent advances in Doppler ultrasound technology permitting measurement of flow velocities within epicardial coronary arteries, this aspect of coronary flow and its possible relation to the generation of ischaemia has received renewed interest.

In the normal situation, blood flow through the left coronary arterial system is biphasic, with the majority of flow occurring in diastole. The contribution of systolic flow is, however, not insignificant. An eloquent series of canine studies by Gregg in the $1960 \mathrm{~s}^{11}$ using volumetric flow catheters demonstrated systolic flow to be of the order of $20-30 \%$ of total left coronary flow at rest. Moreover, it was noted that as total left coronary flow increased during exercise, the ratio of the systolic to diastolic components remained the same or even increased. It was also shown that much of the measured systolic flow represented true intramural flow rather than epicardial flow. It is therefore possible that abnormalities of systolic as well as diastolic coronary flow may have deleterious effects on the metabolic supply and demand of the myocardium.

Although Doppler techniques provide velocity rather than volumetric flow data, important information regarding the systolic and diastolic components of coronary flow can still be obtained. Flow velocity profiles in the left anterior descending artery in patents with aortic stenosis and normal coronary arteries have been examined in several studies using intravascular ultrasound, ${ }^{12}{ }^{13}$ and both trans${\text { oesophageal }{ }^{14} \quad 15}^{15}$ and transthoracic echocardiography. ${ }^{16}$ Certain abnormal velocity profiles have been identified that provide new insights into the mechanism of ischaemia in this group.

SYSTOLIC FLOW

In aortic stenosis, peak systolic flow velocities in the left coronary artery tend to be reduced, with a significant number of patients displaying reversal of flow in systole. ${ }^{12-16}$ The extent of these changes shows good correlation with the pressure gradient across the aortic valve ${ }^{12}$ 
and the systolic left ventricular wall stress index. ${ }^{15}$ Similar changes are not seen in the right coronary artery ${ }^{12}$ nor indeed in patients with left ventricular hypertrophy without aortic valve obstruction. ${ }^{14}$ These changes may therefore relate to the marked discrepancy between left ventricular intramyocardial pressures and coronary perfusion pressure during systole in subjects with significant aortic stenosis.

\section{DIASTOLIC FLOW}

Diastolic flow velocity profiles in aortic stenosis appear to be more heterogeneous and are therefore difficult to interpret. These tend to display rather slower acceleration and more rapid deceleration to and from peak velocity of flow than in normal subjects. ${ }^{12}{ }^{15}$ In addition, while peak diastolic velocities do not seem to change in the same way as systolic velocities in relation to transvalvar gradient, there is some evidence that they are increased in patients who complain of angina symptoms..$^{14}$ The mechanism of these diastolic changes is speculative, but may relate to a high end diastolic pressure (and thus wall tension) decreasing acceleration of flow in diastole by a combination of increased coronary impedance and decreased relative coronary perfusion pressure.

The combination of reduced or absent systolic flow plus increased impedance of diastolic flow to the left ventricle may, on the background of impaired coronary flow reserve, provide the "missing link" in the pathogenesis of angina in aortic stenosis. Furthermore, it has been shown that both the abnormal systolic and diastolic velocity profiles associated with this lesion revert to normal soon after aortic valve replacement, prior to any significant reduction in left ventricular mass. ${ }^{12} 16$

\section{Conclusions}

While left ventricular hypertrophy and reduced coronary flow reserve provide a substrate for myocardial ischaemia in aortic stenosis, other more complex factors such as coronary impedance, left ventricular wall stress, and transaortic pressure difference must also play a major role in the aetiology of angina in this condition. The study of phasic coronary artery flow patterns using Doppler echocardiography has improved our understanding of these mechanisms, but more work needs to be done before their role in aortic stenosis is fully defined. Little is known on the relation between flow abnormalities and symptoms; future studies will need to compare directly matched patients with and without angina. Pharmacological stress echocardiography appears to be safe in aortic stenosis ${ }^{5}$; Doppler analysis of left anterior descending artery flow during stress and its relation to the development of regional wall motion abnormalities and symptoms would provide extremely valuable information. Data on left ventricular diastolic filling parameters in addition to coronary artery flow profiles before and after aortic valve replacement may help elucidate the cause of the diastolic flow abnormalities noted.

The clinical implications of our current knowledge of coronary flow in aortic stenosis in the absence of coronary disease are yet to be firmly established. The development of angina in association with significant aortic stenosis confers a poor prognosis that can be dramatically improved by valve replacement. Surgery is therefore a necessity in this situation irrespective of other findings. However, if it can be shown that the degree of coronary flow abnormality is correlated with prognosis it may be possible to use this information to prioritise symptomatic patients awaiting valve replacement. What are the implications for patients without symptoms? Their prognosis appears to be significantly better (even in the presence of severe stenosis) and it has been argued that completely asymptomatic patients with aortic stenosis should not be operated on until symptoms develop. ${ }^{17}$ It is interesting that coronary flow profiles in asymptomatic patients with aortic stenosis appear to be similar to normal, which would be consistent with the more benign outlook. It is not yet clear whether flow profile changes occur before the development of symptoms. If this is the case it may be possible to use the change in flow profile as an indicator for valve replacement rather than waiting for overt symptoms to appear.

These concepts are of course speculative and much work needs to be done. However, it seems likely that as our understanding of this complex situation increases, coronary flow profile data will establish a useful clinical role in the management of patients with aortic stenosis.

TIMOTHY IRVINE

Regional Cardiothoracic Centre, ANTOINETTE KENNY

Freeman Hospital,

Newcastle upon Tyne NE7 7DN, United Kingdom

1 Morrison GW, Thomas RD, Grimmer SFM, Silverton PN, Smith DR. Incidence of coronary artery disease in patients with valvular heart disease. Br Heart f 1980;44:630-7.

2 Ramsdale DR, Bennett DH, Bray CL, Ward C, Beton DC, Faragher EB. Angina, coronary risk factors and coronary artery disease in patients with valvular heart disease. A prospective study. Eur Heart f 1984;5:716-26.

3 Wood P. Aortic stenosis. Am $\mathcal{F}$ Cardiol 1958;1:553-71.

4 Kupari M, Virtanen KS, Turto H, Viitasalo M, Mänttäri M, Lindroos $M$, et al. Exclusion of coronary artery disease by exercise thallium-201 tomography in patients with aortic valve stenosis. Am f Cardiol 1992;70: 635-40.

5 Baroni M, Maffei S, Terrazzi M, Palmieri C, Paoli F, Biagini A. Mechanisms of regional ischaemic changes during dipyridamole echocardiography in patients with severe aortic valve stenosis and normal cardiography in patients with severe aorti

6 Strauer BE. The concept of coronary flow reserve. 7 Cardiovasc Pharmacol 1992;19(Suppl 5):S67-80

7 Marcus ML, Doty DB, Hiratzka LF, Wright CB, Eastham CL. Decreased coronary reserve: a mechanism for angina pectoris in patients with aortic stenosis and normal coronary arteries. N Engl f Med 1982;307:1362-6

8 Eberli FR, Schwitter J, Bortone A, Schneider J, Hess OM, Krayenbuehl HP. Coronary reserve in patients with aortic valve disease before and after successful aortic valve replacement. Eur Heart $\mathcal{f}$ 1991;12:127-38.

9 Villari B, Hess OM, Moccetti D, Vassalli G, Krayenbuehl HP. Effect of progression of left ventricular hypertrophy on coronary artery dimensions in aortic valve disease. $7 \mathrm{Am}$ Coll Cardiol 1992;20:1073-9.

10 Alyono D, Anderson RW, Parrish DG, Dai XZ, Bache RJ. Alterations of myocardial blood flow associated with experimental canine left ventricular hypertrophy secondary to valvular aortic stenosis. Circ Res 1986;58: $47-57$

11 Gregg DE. Physiology of the coronary circulation. Circulation 1963;27: $1128-37$

12 Yoshikawa J, Akasaka T, Yoshida K, Takagi T. Systolic coronary flow reversal and abnormal diastolic flow patterns in patients with aortic stenosis: assessment with an intracoronary Doppler catheter. $f \mathrm{Am}$ Soc Echocardiogr 1993;6:516-24.

13 Hongo M, Goto T, Watanabe N, Nakatsuka T, Tanaka M, Kinoshita O, et al. Relation of phasic coronary flow velocity profile to clinical and hemodynamic characteristics of patients with aortic valve disease. Circulation dynamic characteri

14 Isaaz $\mathrm{K}$, Bruntz JF, Paris D, Ethevenot G, Aliot E. Abnormal coronary flow velocity pattern in patients with left ventricular hypertrophy, angina pectoris and normal coronary arteries: a transesophageal Doppler echocardiographic study. Am Heart $\mathcal{F}$ 1994;128:500-10

15 Omran H, Fehske W, Rabahieh R, Hagendorff A, Luderitz B. Relation between symptoms and profiles of coronary artery blood flow velocities in patients with aortic valve stenosis: a study using transoesophageal Doppler echocardiography. Heart 1996;75:377-83

16 Kenny A, Wisbey CR, Shapiro LM. Profiles of coronary blood flow velocity in patients with aortic stenosis and the effect of valve replacement: a transthoracic echocardiographic study. Br Heart f 1994;71:57-62.

17 Braunwald E. On the natural history of severe aortic stenosis. $\mathcal{f}$ Am Coll Cardiol 1990;15:1018-20. 